\title{
Agility and Performance in Elastic Distributed Storage
}

\author{
LIANGHONG XU, JAMES CIPAR, ELIE KREVAT, ALEXEY TUMANOV, \\ and NITIN GUPTA, Carnegie Mellon University \\ MICHAEL A. KOZUCH, Intel Labs \\ GREGORY R. GANGER, Carnegie Mellon University
}

\begin{abstract}
Elastic storage systems can be expanded or contracted to meet current demand, allowing servers to be turned off or used for other tasks. However, the usefulness of an elastic distributed storage system is limited by its agility: how quickly it can increase or decrease its number of servers. Due to the large amount of data they must migrate during elastic resizing, state of the art designs usually have to make painful trade-offs among performance, elasticity, and agility.

This article describes the state of the art in elastic storage and a new system, called SpringFS, that can quickly change its number of active servers, while retaining elasticity and performance goals. SpringFS uses a novel technique, termed bounded write offloading, that restricts the set of servers where writes to overloaded servers are redirected. This technique, combined with the read offloading and passive migration policies used in SpringFS, minimizes the work needed before deactivation or activation of servers. Analysis of real-world traces from Hadoop deployments at Facebook and various Cloudera customers and experiments with the SpringFS prototype confirm SpringFS's agility, show that it reduces the amount of data migrated for elastic resizing by up to two orders of magnitude, and show that it cuts the percentage of active servers required by $67-82 \%$, outdoing state-of-the-art designs by $6-120 \%$.
\end{abstract}

Categories and Subject Descriptors: D.4.3 [Operating Systems]: File Systems Management-Distributed file systems

General Terms: Design, Performance

Additional Key Words and Phrases: Cloud storage, distributed file systems, elastic storage, agility, power, write offloading

\section{ACM Reference Format:}

Xu, L., Cipar, J., Krevat, E., Tumanov, A., Gupta, N., Kozuch, M. A., and Ganger, G. R. 2014. Agility and performance in elastic distributed storage. ACM Trans. Storage 10, 4, Article 16 (October 2014), 27 pages.

DOI : http://dx.doi.org/10.1145/2668129

\section{INTRODUCTION}

Distributed storage can and should be elastic, just like other aspects of cloud computing. When storage is provided via single-purpose storage devices or servers, separated from compute activities, elasticity is useful for reducing energy usage, allowing temporarily unneeded storage components to be powered down. However, for storage

An earlier version of this article appeared in Proceedings of the 12th USENIX Conference on File and Storage Technologies (FAST).

This research was sponsored in part by Intel as part of the Intel Science and Technology Center for Cloud Computing (ISTC-CC). Experiments were enabled by generous hardware donations from Intel, NetApp, and APC.

Authors' addresses: L. Xu (corresponding author), A. Tumanov, N. Gupta, and G. Ganger, Electrical and Computer Engineering Department, Carnegie Mellon University; J. Cipar and E. Krevat, Computer Science Department, Carnegie Mellon University; M. Kozuch, Intel Labs, Pittsburgh; corresponding author's email: lianghon@andrew.cmu.edu.

Permission to make digital or hard copies of all or part of this work for personal or classroom use is granted without fee provided that copies are not made or distributed for profit or commercial advantage and that copies bear this notice and the full citation on the first page. Copyrights for components of this work owned by others than ACM must be honored. Abstracting with credit is permitted. To copy otherwise, or republish, to post on servers or to redistribute to lists, requires prior specific permission and/or a fee. Request permissions from permissions@acm.org.

2014 Copyright held by the Owner/Author. Publication rights licensed to ACM. 1553-3077/2014/10-ART16 $\$ 15.00$

DOI : http://dx.doi.org/10.1145/2668129 
provided via multi-purpose servers (e.g., when a server operates as both a storage node in a distributed filesystem and a compute node), such elasticity is even more valuable-providing cloud infrastructures with the freedom to use such servers for other purposes, as tenant demands and priorities dictate. This freedom may be particularly important for increasingly prevalent data-intensive computing activities (e.g., data analytics).

Data-intensive computing over big datasets is quickly becoming important in most domains and will be a major consumer of future cloud computing resources [AMPLab 2013; Bryant 2007; Hardesty 2012; ISTC-CC 2013]. Many of the frameworks for such computing (e.g., Hadoop [2012] and Google's MapReduce [Dean and Ghemawat 2008]) achieve efficiency by distributing and storing the data on the same servers used for processing it. Usually, the data is replicated and spread evenly (via randomness) across the servers, and the entire set of servers is assumed to always be part of the data analytics cluster. Little-to-no support is provided for elastic sizing $^{1}$ of the portion of the cluster that hosts storage-only nodes that host no storage can be removed without significant effort, meaning that the storage service size can only grow.

Some recent distributed storage designs (e.g., Sierra [Thereska et al. 2011], Rabbit [Amur et al. 2010]) provide for elastic sizing, originally targeted for energy savings, by distributing replicas among servers such that subsets of them can be powered down when the workload is low without affecting data availability; any server with the primary replica of data will remain active. These systems are designed mainly for performance or elasticity (how small the system size can shrink to) goals, while overlooking the importance of agility (how quickly the system can resize its footprint in response to workload variations), which we find has a significant impact on the machine-hour savings (and so the operating cost savings) one can potentially achieve. As a result, state-of-the-art elastic storage systems must make painful tradeoffs among these goals, unable to fulfill them at the same time. For example, Sierra balances load across all active servers and thus provides good performance. However, this even data layout limits elasticity - at least one third of the servers must always be active (assuming 3-way replication), wasting machine hours that could be used for other purposes when the workload is very low. Further, rebalancing the data layout when turning servers back on induces significant migration overhead, impairing system agility.

In contrast, Rabbit can shrink its active footprint to a much smaller size $(\approx 10 \%$ of the cluster size), but its reliance on Everest-style write offloading [Narayanan et al. 2008 b] induces significant cleanup overhead when shrinking the active server set, resulting in poor agility.

This article describes a new elastic distributed storage system, called SpringFS, that provides the elasticity of Rabbit and the peak write bandwidth characteristic of Sierra, while maximizing agility at each point along a continuum between their respective best cases. The key idea is to employ a small set of servers to store all primary replicas nominally, but (when needed) offload writes that would go to overloaded servers to only the minimum set of servers that can satisfy the write throughput requirement (instead of all active servers). This technique, termed bounded write offloading, effectively restricts the distribution of primary replicas during offloading and enables SpringFS to adapt dynamically to workload variations while meeting performance targets with a

\footnotetext{
${ }^{1}$ We use "elastic sizing" to refer to dynamic online resizing, down from the full set of servers and back up, such as to adapt to workload variations. The ability to add new servers, as an infrequent administrative action, is common but does not itself make a storage service "elastic" in this context; likewise with the ability to survive failures of individual storage servers.
} 
minimum loss of agility-most of the servers can be extracted without needing any pre-removal cleanup. SpringFS further improves agility by minimizing the cleanup work involved in resizing with two more techniques: read offloading offloads reads from write-heavy servers to reduce the amount of write offloading needed to achieve the system's performance targets; passive migration delays migration work by a certain time threshold during server re-integration to reduce the overall amount of data migrated. With these techniques, SpringFS achieves agile elasticity while providing performance comparable to a non-elastic storage system.

Our experiments demonstrate that the SpringFS design enables significant reductions in both the fraction of servers that need to be active and the amount of migration work required. Indeed, its design for where and when to offload writes enables SpringFS to resize elastically without performing any data migration at all in most cases. Analysis of traces from six real Hadoop deployments at Facebook and various Cloudera customers show the oft-noted workload variation and the potential of SpringFS to exploit it-SpringFS reduces the amount of data migrated for elastic resizing by up to two orders of magnitude and cuts the percentage of active servers required by $67-82 \%$, outdoing state-of-the-art designs like Sierra and Rabbit by 6-120\%.

This article makes three main contributions: First, it shows the importance of agility in elastic distributed storage, highlighting the need to resize quickly (at times) rather than just hourly as in previous designs. Second, SpringFS introduces a novel write offloading policy that bounds the set of servers to which writes to over-loaded primary servers are redirected. Bounded write offloading, together with read offloading and passive migration, significantly improves the system's agility by reducing the cleanup work during elastic resizing. These techniques apply generally to elastic storage with an uneven data layout. Third, we demonstrate the significant machine-hour savings that can be achieved with elastic resizing, using six real-world HDFS traces, and the effectiveness of SpringFS's policies at achieving a "close-to-ideal" machine-hour usage.

The remainder of this article is organized as follows. Section 2 describes elastic distributed storage generally, the Rabbit design specifically, the importance of agility in elastic storage, and the limitations of the state-of-the-art data layout designs in fulfilling elasticity, agility, and performance goals at the same time. Section 3 describes SpringFS, including its key techniques for increasing agility of elasticity, support for per-dataset bandwidth control, and fault tolerance. Section 4 overviews the SpringFS implementation. Section 5 evaluates the SpringFS design.

\section{BACKGROUND AND MOTIVATION}

This section motivates our work. First, it describes the related work on elastic distributed storage, which provides different mechanisms and data layouts to allow servers to be extracted while maintaining data availability. Second, it gives additional details on the data layout of one specific instance (Rabbit). Third, it demonstrates the significant impact of agility on aggregate machine-hour usage of elastic storage. At last, it describes the limitations of state-of-the-art elastic storage systems and how SpringFS fills the significant gap between agility and performance.

\subsection{Related Work}

Most distributed storage is not elastic. For example, the cluster-based storage systems commonly used in support of cloud and data-intensive computing environments, such as the Google File System(GFS) [Ghemawat et al. 2003] or the Hadoop Distributed Filesystem [Hadoop 2012], use data layouts that are not amenable to elasticity. The Hadoop Distributed File System (HDFS), for example, uses a replication and data-layout policy wherein the first replica is placed on a node in the same rack as the writing node (preferably the writing node, if it contributes to DFS storage), the 
second and third on random nodes in a randomly chosen different rack than the writing node. In addition to load balancing, this data layout provides excellent availability properties-if the node with the primary replica fails, the other replicas maintain data availability; if an entire rack fails (e.g., through the failure of a communication link), data availability is maintained via the replica(s) in another rack. But, such a data layout prevents elasticity by requiring that almost all nodes be active-no more than one node per rack can be turned off without a high likelihood of making some data unavailable.

Recent research [Amur et al. 2010; Leverich and Kozyrakis 2009; Saito et al. 2004; Thereska et al. 2011; Vasić et al. 2009] has provided new data layouts and mechanisms for enabling elasticity in distributed storage. Most notable are Rabbit [Amur et al. 2010] and Sierra [Thereska et al. 2011]. Both organize replicas such that one copy of data is always on a specific subset of servers, termed primaries, so as to allow the remainder of the nodes to be powered down without affecting availability, when the workload is low. With workload increase, they can be turned back on. The same designs and data distribution schemes would allow for servers to be used for other functions, rather than turned off, such as for higher-priority (or higher paying) tenants' activities. Writes intended for servers that are inactive ${ }^{2}$ are instead written to other active servers-an action called write availability offloading-and then later reorganized (when servers become active) to conform to the desired data layout.

Rabbit and Sierra build on a number of techniques from previous systems, such as write availability offloading and power gears. Narayanan et al. [2008a] described the use of write availability offloading for power management in enterprise storage workloads. The approach was used to redirect traffic from otherwise idle disks to increase periods of idleness, allowing the disks to be spun down to save power. PARAID [Weddle et al. 2007] introduced a geared scheme to allow individual disks in a RAID array to be turned off, allowing the power used by the array to be proportional to its throughput.

Everest [Narayanan et al. 2008b] is a distributed storage design that used write performance offloading ${ }^{3}$ in the context of enterprise storage. In Everest, disks are grouped into distinct volumes, and each write is directed to a particular volume. When a volume becomes overloaded, writes can be temporarily redirected to other volumes that have spare bandwidth, leaving the overloaded volume to only handle reads. Rabbit applies this same approach, when necessary, to address overload of the primaries.

SpringFS borrows the ideas of write availability and performance offloading from prior elastic storage systems. Specifically, it builds on the Rabbit design but develops new offloading and migration schemes that effectively eliminate the painful trade-off between agility and write performance. These techniques apply generally to elastic storage designs with an uneven data layout.

\subsection{Rabbit Data Layout}

Rabbit [Amur et al. 2010] is a distributed file system designed to provide power proportionality to workloads that vary over time. It is based on HDFS [Borthakur 2007] but uses alternate data layouts that allow it to extract (e.g., power off) large subsets of servers without reducing data availability. To do so, Rabbit exploits the data replicas

\footnotetext{
${ }^{2}$ We generally refer to a server as inactive when it is either powered down or reused for other purposes. Conversely, we call a server active when it is powered on and servicing requests as part of a elastic distributed storage system.

${ }^{3}$ Write performance offloading differs from write availability offloading in that it offloads writes from overloaded active servers to other (relatively idle) active servers for better load balancing. The Everest-style and bounded write offloading schemes are both types of write performance offloading.
} 
(originally for fault tolerance) to ensure that all blocks are available at any power setting. In addition to maintaining availability, Rabbit tries to be power proportional with any number of active servers, that is, performance scales linearly with the number of active servers. This section describes in detail the equal-work data layout that Rabbit relies on to achieve power proportionality.

Consider a cluster with $N$ nodes, where $t_{p u t}$ is the I/O throughput obtained and pow $_{n}$ the power consumed when $n$ nodes are active (powered on). The requirements of a power-proportional distributed storage system is formally stated here.

(1) A low minimum throughput, $t_{p} t_{p}$, consuming power pow $_{p}$, where $p$ nodes are kept active and $p \ll N$.

(2) A high maximum throughput, tput $_{N}$, consuming power pow ${ }_{N}$, when $N$ nodes are kept active.

(3) Ideal power-proportionality, which means that $t p u t_{i} /$ pow $_{i}=t p u t_{N} /$ pow $_{N}$ for any $i \in\{p, \ldots, N\}$.

(4) Fast, fine-grained scaling with no data movement required.

The equal-work policy is the result of an optimization problem that minimizes $p$ with the constraints, tput $_{i}=(i / p) t p u t_{p}$ for all $i=p+1, \ldots, N$ for a given replication factor $r$. The following sections offer an intuitive explanation of the equal-work policy. An example is shown in Figure 1 for the case of $N=100, B=10^{4}, p=5$, and $r=4$.

2.2.1. Definitions. A dataset is an arbitrary user-defined set of files stored in the distributed file system (DFS). For each dataset, we define an ordered list of nodes, called the expansion-chain, which denotes the order in which nodes must be turned on or off to scale performance up or down, respectively. The nodes of the expansion-chain that are powered on are called the active nodes, $A(d)$, for dataset $d$. For the rest of Section 2.2, we do not consider multiple datasets, which will be discussed further in Section 3.3.

2.2.2. Low Minimum Power. In the equal-work data-layout policy, the first $p$ nodes of the expansion-chain are called the primary nodes. One replica of the dataset, called the primary replica, is distributed evenly over the primary nodes as shown in Figure 1. Keeping only these $p$ nodes, on is sufficient for guaranteeing the availability of all data. Because $p \ll N$, this gives Rabbit a low minimum power setting.

2.2.3. Ideal Power-Proportionality. To ensure ideal power-proportionality, $b_{i}=B / i$ blocks are stored on the $i$ th node of the expansion-chain, where $i>p$. This satisfies a necessary condition for ideal power-proportionality that is violated by the naïve policy, which is that $b_{i}$, the number of blocks stored by $i$ th node in the expansion-chain, must not be less than $B / n$ for all $i \leq n$, when $n$ nodes are active. Obeying this constraint makes it possible for the load to be shared equally among the nodes that are active. To illustrate, consider the situation when an entire dataset of $B$ blocks has to be read from the DFS with $n \geq p$ nodes active. For ideal power-proportionality, each of the nodes should service $B / n$ blocks. This is made possible by the equal-work layout, because the $n$th node stores $B / n$ blocks, and each of the nodes $i$ with $i \in[p, n)$ stores $B / i>B / n$ blocks. To scale performance up, the number of active nodes is increased by turning on nodes according to the order specified by the expansion-chain for the dataset. Scaling requires no data movement and can be done at the granularity of a single node.

2.2.4. High Maximum Performance Setting. Each node stores no more than the minimum required number of blocks, which allows the blocks to be distributed across a larger number of nodes while holding the number of replicas fixed so energy is not wasted 


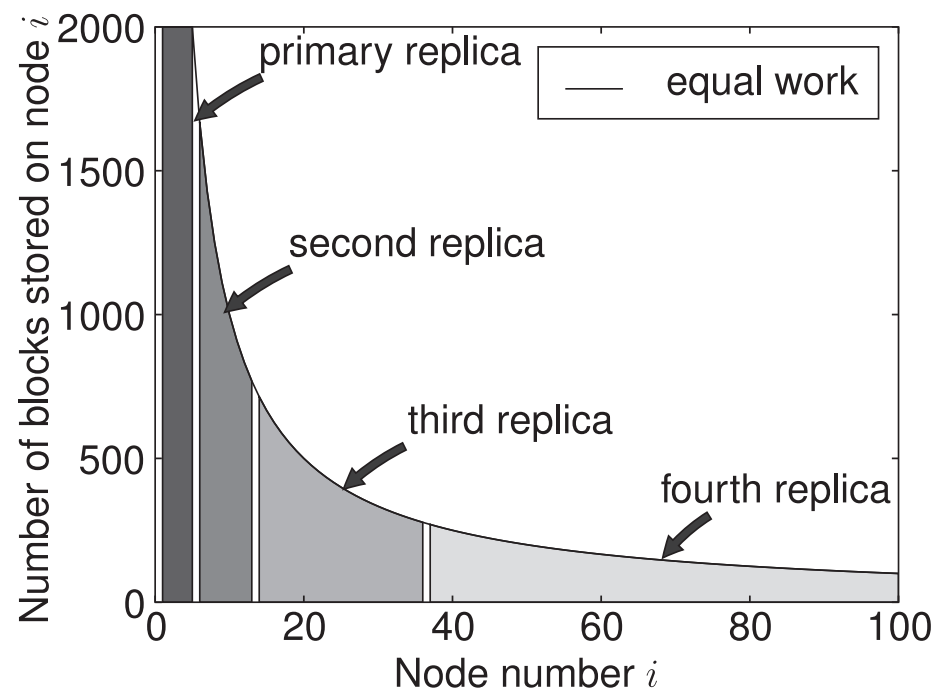

Fig. 1. Equal-work data layout.

writing an unnecessarily high number of data copies. We define a dataset's spread to be the number of nodes over which the blocks of that dataset are stored. A dataset's spread is equal to the length of its expansion-chain. For the equal-work policy, the spread depends on the number of replicas used.

We can derive a lower bound on the spread based on the observation that the number of blocks stored on the servers in the range $[p+1, s]$ must correspond to $(r-1)$ replicas of the dataset. Hence,

$$
\sum_{i=p+1}^{s} B / i=B(r-1) .
$$

Because $1 / i$ is a monotonically-decreasing function, we also have convenient lower and upper bounds on $\sum_{i=p+1}^{s} 1 / i$ as

$$
\int_{p+1}^{s+1}(1 / x) d x \leq \sum_{i=p+1}^{s} 1 / i \leq \int_{p}^{s}(1 / x) d x .
$$

From Equations (1) and (2), we get

$$
s \geq p e^{r-1} .
$$

Note that the spread increases exponentially with the number of replicas while maintaining ideal power-proportionality. Since the maximum throughput obtainable depends on the spread, this allows the equal-work policy to obtain a high value for the same. We note that, since the spread also depends on $p$, a spread spanning the entire cluster can be obtained with any number of replicas $r$ by adjusting the value of $p$.

\subsection{Agility Is Important}

By "agility," we mean how quickly one can change the number of servers effectively contributing to a service. For most non-storage services, such changes can often be 


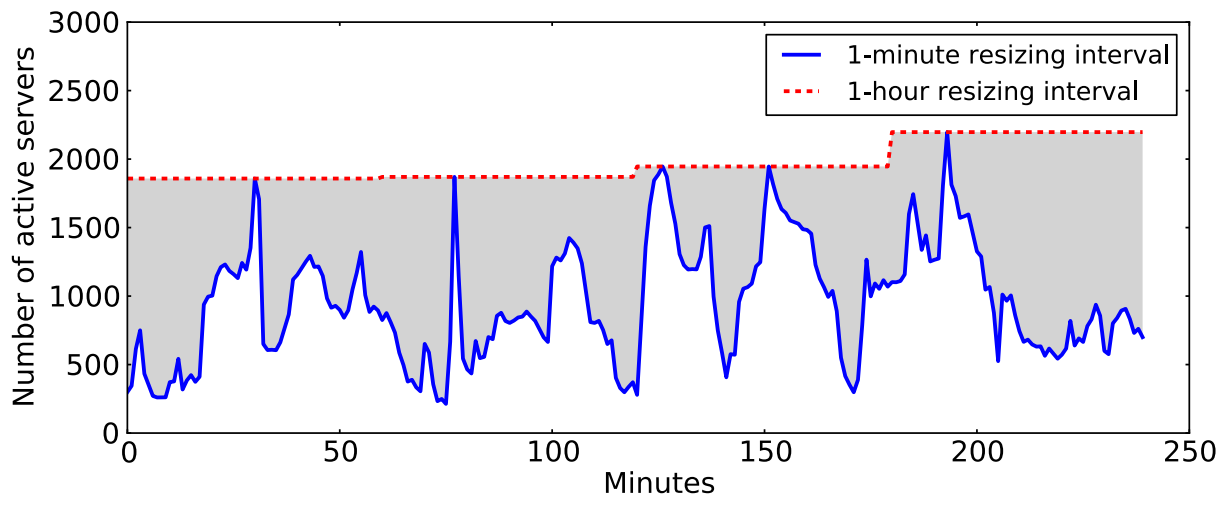

Fig. 2. Workload variation in the Facebook trace. The shaded region represents the potential reduction in machine-hour usage with a 1-minute resizing interval.

completed quickly, as the amount of state involved is small. For distributed storage, however, the state involved may be substantial. A storage server can service reads only for data that it stores, which affects the speed of both removing and reintegrating a server. Removing a server requires first ensuring that all data is available on other servers, and reintegrating a server involves replacing data overwritten (or discarded) while it was inactive.

The time required for such migrations has a direct impact on the machine hours consumed by elastic storage systems. Systems with better agility are able to more effectively exploit the potential of workload variation by more closely tracking workload changes. Previous elastic storage systems rely on very infrequent changes (e.g., hourly resizing in Sierra [Thereska et al. 2011]), but we find that over half of the potential savings is lost with such an approach due to the burstiness of real workloads.

As one concrete example, Figure 2 shows the number of active servers needed, as a function of time in the trace, to provide the required throughput in a randomly chosen 4-hour period from the Facebook trace described in Section 5. The dashed and solid curves bounding the shaded region represent the minimum number of active servers needed if using 1-hour and 1-minute resizing intervals, respectively. For each such period, the number of active servers corresponds to the number needed to provide the peak throughput in that period, as is done in Sierra to avoid significant latency increases. The area under each curve represents the machine time used for that resizing interval, and the shaded region represents the increased server usage (more than double) for the 1-hour interval. We observe similar burstiness and consequences of it across all of the traces.

\subsection{Bridging Agility and Performance}

Previous elastic storage systems overlook the importance of agility, focusing on performance and elasticity. This section describes the limitation of the data layouts of state-of-the-art elastic storage systems, specifically Sierra and Rabbit, and how their layouts represent two specific points in the trade-off space among elasticity, agility, and performance. Doing so highlights the need for a more flexible elastic storage design that fills the void between them, providing greater agility and matching the best of each.

We focus on elastic storage systems that ensure data availability at all times. When servers are extracted from the system, at least one copy of all data must remain active to serve read requests. Like Rabbit, Sierra also exploits data replicas to ensure that all 


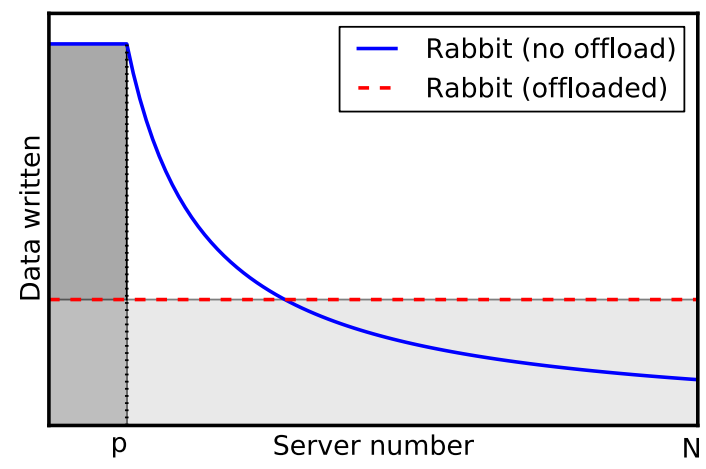

Fig. 3. Primary data distribution for Rabbit without offloading (grey) and Rabbit with offloading (light grey). With offloading, primary replicas are spread across all active servers during writes, incurring significant cleanup overhead when the system shrinks its size.

blocks are available at any power setting. With 3 -way replication ${ }^{4}$, Sierra stores the first replica of every block (termed primary replica) in one third of servers, and writes the other two replicas to the other two thirds of servers. This data layout allows Sierra to achieve full peak performance due to balanced load across all active servers, but it limits the elasticity of the system by not allowing the system footprint to go below one third of the cluster size. We show in Section 5.2 that such limitation can have a significant impact on the machine-hour savings that Sierra can potentially achieve, especially during periods of low demand.

Rabbit, on the other hand, is able to reduce its system footprint to a much smaller size (down to $p$ ) without any cleanup work in virtue of the equal-work data layout. However, it can create bottlenecks for writes. Since the primary servers must store the primary replicas for all blocks, the maximum write throughput of Rabbit is limited by the maximum aggregate write throughout of the $p$ primary servers, even when all servers are active. In contrast, Sierra is able to achieve the same maximum write throughput as that of HDFS, that is, the aggregate write throughput of $N / 3$ servers (recall: $N$ servers write three replicas for every data block).

Rabbit borrows write offloading from the Everest system [Narayanan et al. 2008b] to solve this problem. When primary servers become the write performance bottleneck, Rabbit simply offloads writes that would go to heavily loaded servers across all active servers. While such write offloading allows Rabbit to achieve good peak write performance comparable to unmodified HDFS due to balanced load, it significantly impairs system agility by spreading primary replicas across all active servers, as depicted in Figure 3. Consequently, before Rabbit shrinks the system size, cleanup work is required to migrate some primary replicas to the remaining active servers so that at least one complete copy of data is still available after the resizing action. As a result, the improved performance from Everest-style write offloading comes at a high cost in system agility.

Figure 4 illustrates the very different design points represented by Sierra and Rabbit, in terms of the trade-offs among agility, elasticity, and peak write performance. Read performance is the same for all of these systems, given the same number of active servers. The minimum number of active servers that store primary replicas

\footnotetext{
${ }^{4}$ For simplicity, we assume 3-way replication for all data blocks in the rest of this article, which remains the default policy for HDFS. The data layout designs apply to other replication levels as well. Different approaches than Sierra, Rabbit, and SpringFS are needed when erasure codes are used for fault tolerance instead of replication.
} 


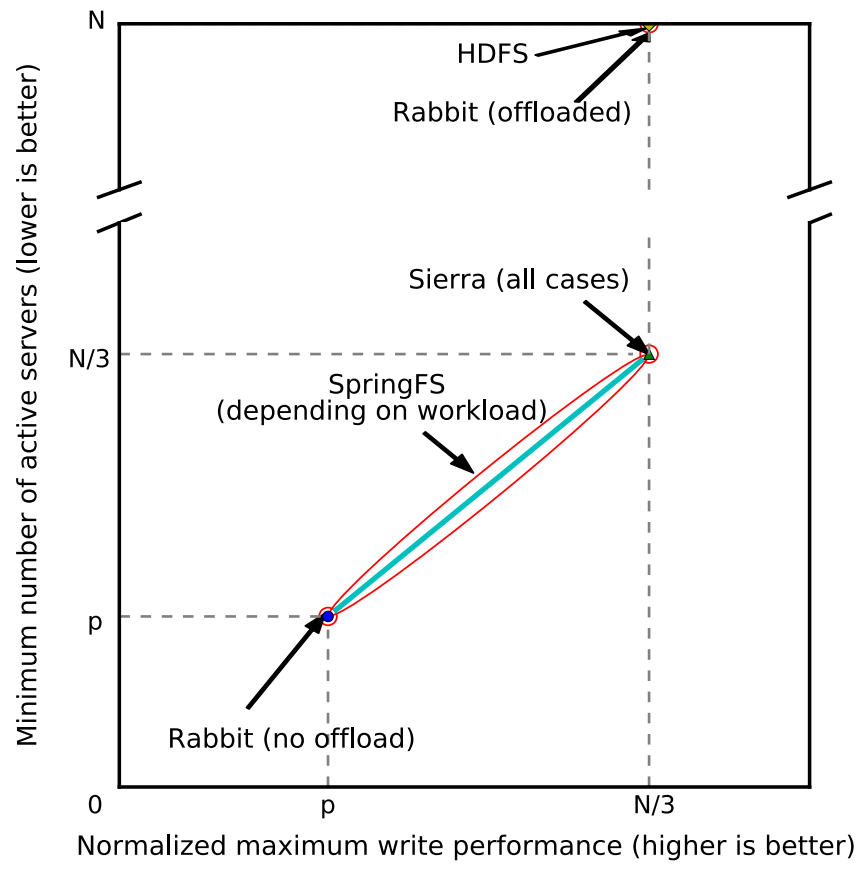

Fig. 4. Elastic storage system comparison in terms of agility and performance. $N$ is the total size of the cluster. $p$ is the number of primary servers in the equal-work data layout. Servers with at least some primary replicas cannot be deactivated without first moving those primary replicas. SpringFS provides a continuum between Sierra's and Rabbit's (when no offload) single points in this trade-off space. When Rabbit requires offload, SpringFS is superior at all points. Note that the y-axis is discontinuous.

indicates the minimal system footprint one can shrink to without any cleanup work. The maximum write performance shown on the x-axis is normalized to the throughput of a single server. As described in the preceding text, state-of-the art elastic storage systems such as Sierra and Rabbit suffer from the painful trade-off between agility and performance due to the use of a rigid data layout. SpringFS provides a more flexible design that provides the best-case elasticity of Rabbit, the best-case write performance of Sierra, and much better agility than either. To achieve the range of options shown, SpringFS uses an explicit bound on the offload set, where writes of primary replicas to overloaded servers are offloaded to only the minimum set of servers (instead of all active servers) that can satisfy the current write throughput requirement. This additional degree of freedom allows SpringFS to adapt dynamically to workload changes, providing the desired performance while maintaining system agility.

\section{SPRINGFS DESIGN AND POLICIES}

This section describes SpringFS's data layout as well as the bounded write offloading and read offloading policies that minimize the cleanup work needed before deactivation of servers. It then describes the passive migration policy used during a server's reintegration to address data that was written during the server's absence. Lastly, it presents the I/O scheduling and fault tolerance mechanisms used in both Rabbit and SpringFS. ${ }^{5}$

\footnotetext{
${ }^{5}$ In principle, the I/O scheduling and fault-tolerance mechanisms in Rabbit and SpringFS are the same, because both systems are based on the equal-work data layout. For simplicity in illustration, we use Rabbit (without write offloading) as an example.
} 


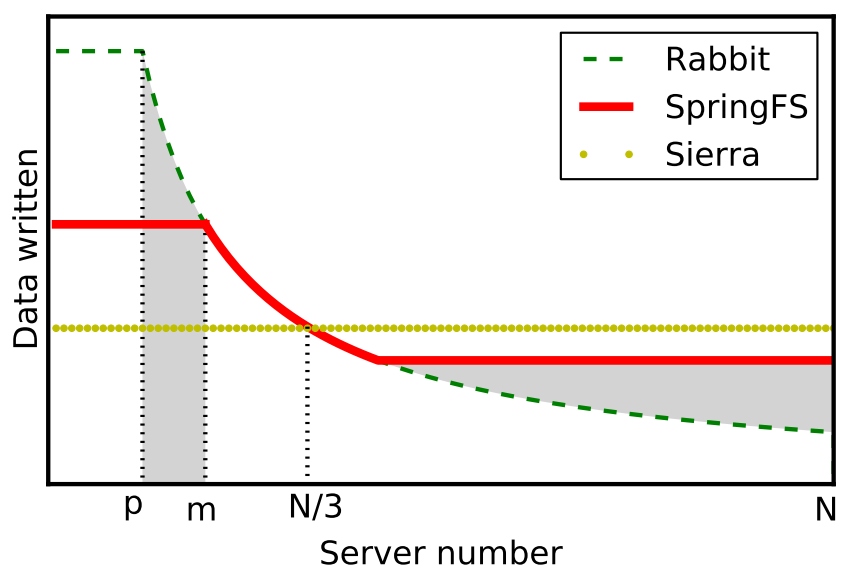

Fig. 5. SpringFS data layout and its relationship with previous designs. The offload set allows SpringFS to achieve a dynamic trade-off between the maximum write performance and the cleanup work needed before extracting servers. In SpringFS, all primary replicas are stored in the $m$ servers of the offload set. The shaded regions indicate writes of non-primary replicas that would have gone to the offload set (in SpringFS) are instead redirected and load balanced outside the set.

\subsection{Data Layout and Offloading Policies}

Data Layout. Regardless of write performance, the equal-work data layout proposed in Rabbit enables the smallest number of primary servers and thus provides the best elasticity in state-of-the-art designs. ${ }^{6}$ SpringFS retains such elasticity using a variant of the equal-work data layout, but addresses the agility issue incurred by Everest-style offloading when write performance bottlenecks arise. The key idea is to bound the distribution of primary replicas to a minimal set of servers (instead of offloading them to all active servers), given a target maximum write performance, so that the cleanup work during server extraction can be minimized. This bounded write offloading technique introduces a parameter called the offload set: the set of servers to which primary replicas are offloaded (and as a consequence receive the most write requests). The offload set provides an adjustable trade-off between maximum write performance and cleanup work. With a small offload set, few writes will be offloaded, and little cleanup work will be subsequently required, but the maximum write performance will be limited. Conversely, a larger offload set will offload more writes, enabling higher maximum write performance at the cost of more cleanup work to be done later. Figure 5 shows the SpringFS data layout and its relationship with the state-of-the-art elastic data layout designs. We denote the size of the offload set as $m$, the number of primary servers in the equal-work layout as $p$, and the total size of the cluster as $N$. When $m$ equals $p$, SpringFS behaves like Rabbit and writes all data according to the equalwork layout (no offload); when $m$ equals $N / 3$, SpringFS behaves like Sierra and load balances all writes (maximum performance). As illustrated in Figure 4, the use of the tunable offload set allows SpringFS to achieve both end points and points in between.

Choosing the Offload Set. The offload set is not a rigid setting but determined on the fly to adapt to workload changes. Essentially, it is chosen according to the target maximum write performance identified for each resizing interval. Because servers in the offload set write one complete copy of the primary replicas, the size of the offload set is simply the maximum write throughput in the workload divided by the write

\footnotetext{
${ }^{6}$ Theoretically, no other data layout can achieve a smaller number of primary servers while maintaining power-proportionality for read performance.
} 
throughput a single server can provide. Section 5.2 gives a more detailed description of how SpringFS chooses the offload set (and the number of active servers) given the target workload performance.

Read Offloading. One way to reduce the amount of cleanup work is to simply reduce the amount of write offloading that needs to be done to achieve the system's performance targets. When applications simultaneously read and write data, SpringFS can coordinate the read and write requests so that reads are preferentially sent to higher numbered servers that naturally handle fewer write requests. We call this technique read offloading.

Despite its simplicity, read offloading allows SpringFS to increase write throughput without changing the offload set by taking read work away from the low numbered servers (which are the bottleneck for writes). When a read occurs, instead of randomly picking one among the servers storing the replicas, SpringFS chooses the server that has received the least number of total requests recently. (The one exception is when the client requesting the read has a local copy of the data. In this case, SpringFS reads the replica directly from that server to exploit machine locality.) As a result, lower-numbered servers receive more writes, while higher-numbered servers handle more reads. Such read/write distribution balances the overall load across all the active servers while reducing the need for write offloading.

Replica Placement. When a block write occurs, SpringFS chooses target servers for the three replicas in the following steps: The primary replica is load balanced across (and thus bounded in) the $m$ servers in the current offload set. (The one exception is when the client requesting the write is in the offload set. In this case, SpringFS writes the primary copy to that server, instead of the server with the least load in the offload set, to exploit machine locality.) For non-primary replicas, SpringFS first determines their target servers according to the equal-work layout. For example, the target server for the secondary replica would be a server numbered between $p+1$ and $e p$, and that for the tertiary replica would be a server numbered between $e p+1$ and $e^{2} p$, both following the probability distribution as indicated by the equal-work layout (lower numbered servers have higher probability to write the non-primary replicas). If the target server number is higher than $m$, the replica is written to that server. However, if the target server number is between $p+1$ and $m$ (a subset of the offload set), the replica is instead redirected and load balanced across servers outside the offload set, as shown in the shaded regions in Figure 5. Such redirection of non-primary replicas reduces the write requests going to the servers in the offload set and ensures that these servers store only the primary replicas.

\subsection{Passive Migration for Reintegration}

When SpringFS tries to write a replica according to its target data layout but the chosen server happens to be inactive, it must still maintain the specified replication factor for the block. To do this, another host must be selected to receive the write. Availability offloading is used to redirect writes that would have gone to inactive servers (which are unavailable to receive requests) to the active servers. As illustrated in Figure 6, SpringFS load balances availability offloaded writes together with the other writes to the system. This results in the availability offloaded writes going to the less-loaded active servers rather than adding to existing write bottlenecks on other servers.

Because of availability offloading, reintegrating a previously deactivated server is more than simply restarting its software. While the server can begin servicing its share of the write workload immediately, it can only service reads for blocks that it stores. 


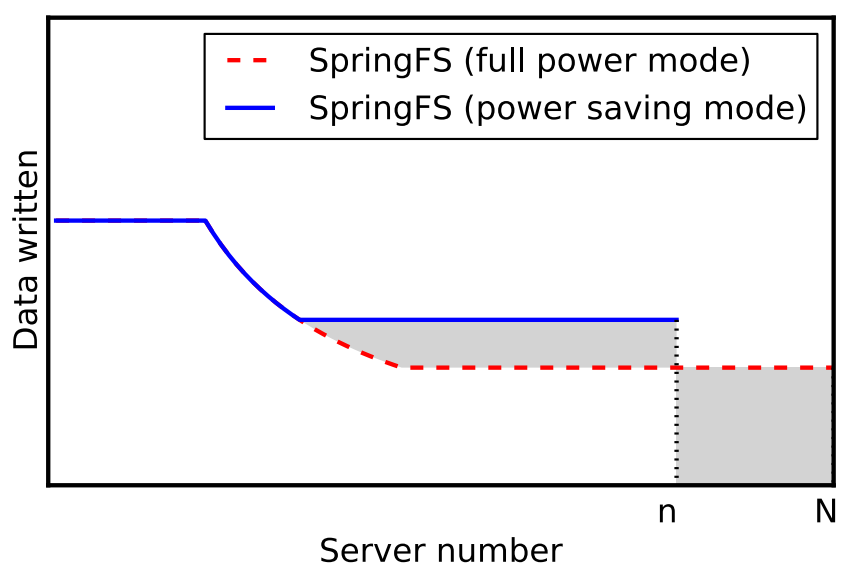

Fig. 6. Availability offloading. When SpringFS works in the power saving mode, some servers $(n+1$ to $N)$ are deactivated. The shaded regions show that writes that would have gone to these inactive servers are offloaded to higher numbered active servers for load balancing.

Thus, filling it according to its place in the target equal-work layout is part of full reintegration.

When a server is reintegrated to address a workload increase, the system needs to make sure that the active servers will be able to satisfy the read performance requirement. One option is to aggressively restore the equal work data layout before reintegrated servers begin servicing reads. We call this approach aggressive migration. Before anticipated workload increases, the migration agent would activate the right number of servers and migrate some data to the newly activated servers so that they store enough data to contribute their full share of read performance. The migration time is determined by the number of blocks that need to be migrated, the number of servers that are newly activated, and the I/O throughput of a single server. With aggressive migration, cleanup work is never delayed. Whenever a resizing action takes place, the property of the equal-work layout is obeyed-server $x$ stores no less than $\frac{B}{x}$ blocks.

SpringFS takes an alternate approach called passive migration, based on the observation that cleanup work when reintegrating a server is not as important as when deactivating a server (for which it preserves data availability), and that the total amount of cleanup work can be reduced by delaying some fraction of migration work while performance goals are still maintained (which makes this approach better than aggressive migration). Instead of aggressively fixing the data layout (by activating the target number of servers in advance for a longer period of time), SpringFS temporarily activates more servers than would minimally be needed to satisfy the read throughput requirement and utilizes the extra bandwidth for migration work and to address the reduced number of blocks initially on each reactivated server. The number of extra servers that need to be activated is determined in two steps. First, an initial number is chosen to ensure that the number of valid data blocks still stored on the activated servers is more than the fraction of read workload they need to satisfy so that the performance requirement is satisfied. Second, the number may be increased so that the extra servers provide enough I/O bandwidth to finish a fraction $(1 / T$, where $\mathrm{T}$ is the migration threshold as described below) of migration work. To avoid migration work building up indefinitely, the migration agent sets a time threshold so that whenever a migration action takes place, it is guaranteed to finish within $T$ minutes. With $T>1$ (the default resizing interval), SpringFS delays part of the migration work while 
satisfying throughput requirement. Because higher numbered servers receive more writes than their equal-work share, due to write offloading, some delayed migration work can be replaced by future writes, which reduces the overall amount of data migration. If $\mathrm{T}$ is too large, however, the cleanup work can build up so quickly that even activating all the servers cannot satisfy the throughput requirement. In practice, we find a migration threshold $T=10$ to be a good choice and use this setting for the trace analysis in Section 5. Exploring automatic setting of $\mathrm{T}$ is an interesting future work.

\subsection{I/O Scheduling with Multi-Dataset Support}

The ability to allocate I/O bandwidth available through the DFS to specific applications that run on it would have significant benefits. Previous results [Isard et al. 2009] show that almost $5 \%$ of the jobs observed in a large-scale data center run for more than five hours and some jobs run for more than a day. In the presence of such long-running jobs, it is imperative to be able to guarantee some notion of fair sharing of the resources of the cluster. There should be capabilities, for example, to temporarily decrease the performance of long jobs during times of high load or when there are higher-priority, shorter-running jobs to be processed. Although Hadoop or an equivalent implementation of the map-reduce paradigm has its own scheduler, the underlying DFS will most likely support multiple kinds of applications in the data center. For example, Google's BigTable [Chang et al. 2008] and Hadoop's HBase are designed to work directly on top of the DFS. It is not possible, with current solutions, to guarantee I/O performance for each of these jobs. In other words, there is no check on a single job monopolizing the $\mathrm{I} / \mathrm{O}$ resources of the cluster. This problem is often exacerbated by the fact that jobs are increasingly data-intensive, such that their overall performance depends significantly on the amount of $\mathrm{I} / \mathrm{O}$ bandwidth that they receive.

The DFS is an ideal location for the implementation of mechanisms to control the amount of bandwidth provisioned to applications. Rabbit and SpringFS can manage $\mathrm{I} / \mathrm{O}$ resources between datasets stored in the cluster. It is possible to allocate $\mathrm{I} / \mathrm{O}$ bandwidth to a particular dataset that would then be shared by the applications using that dataset. This section describes how we use the mechanisms used to provide powerproportionality to perform I/O scheduling for datasets in Rabbit or SpringFS. Recall that a dataset is defined to be an arbitrary set of files stored in Rabbit. We assume that all data entering the system is tagged with metadata specifying the dataset that the data belongs to. One way to do this is to define datasets based on file system hierarchy, with subtrees explicitly associated with datasets, as with volumes in AFS [Zayas 1991].

Our focus is on data-intensive jobs whose performance significantly depends on I/O bandwidth, such as most jobs run on Hadoop. Hadoop has its own fair scheduler that indirectly manages I/O resources by controlling the compute scheduling, but this approach only guarantees fairness for map-reduce jobs using the particular instance of the Hadoop library. In a data center environment, there can exist multiple different applications, such as BigTable [Chang et al. 2008], that use the services offered by the DFS. In such scenarios, indirectly managing the I/O resources through compute scheduling becomes impossible. Our solution enables scheduling of $\mathrm{I} / \mathrm{O}$ resources at the level of the DFS and allows the I/O bandwidth of the cluster to be shared among the datasets in an explicit manner.

Section 2.2 explains the equal-work data layout policy. To handle multiple datasets, we use the same policy but overlay the datasets over one another using a greedy strategy to choose the nodes. We define a score, $s_{i}$ for a node $i$ that depends on where that node figures in the expansion-chains of the different datasets. Let $D_{i}$ be the set of datasets that have blocks stored on node $i$, let $s_{i}(d)$ be the contribution of dataset 


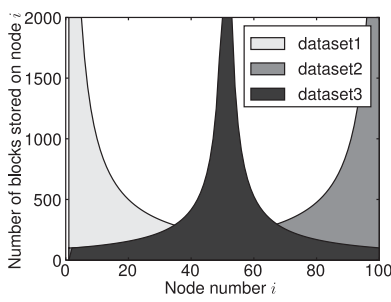

(a) Data layout for multiple datasets.

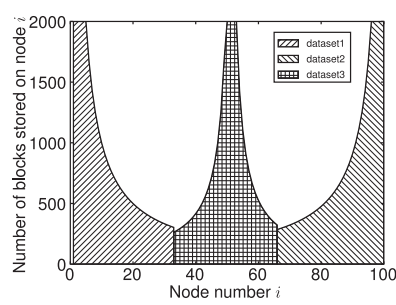

(b) Fair sharing with three live datasets. Shaded regions represent the active nodes.

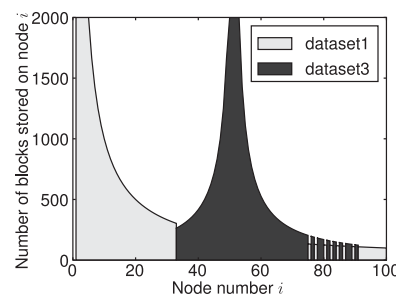

(c) Fair sharing with two live datasets. Shaded regions represent the active nodes.

Fig. 7. Example multi-dataset layout involving three datasets.

$d \in D_{i}$ to the score $s_{i}$, and let $l_{i}(d)$ be the index of node $i$ in the expansion-chain of dataset $d$ where node $i$ appears. Then,

$$
\begin{gathered}
s_{i}=\sum_{d \in D_{i}} s_{i}(d), \\
s_{i}(d)= \begin{cases}1 & \text { if } l_{i}(d) \leq p, \\
\frac{1}{l_{i}(d)} & \text { otherwise } .\end{cases}
\end{gathered}
$$

When a new dataset is to be written into the DFS, nodes are chosen greedily starting with the node with the minimum score, $s_{i}$. The score's are updated once the blocks of the new dataset are stored. Figure 7 shows the layout policy for three datasets. Each of the datasets has a spread that is equal to the size of the entire cluster, but the order of nodes in the expansion-chains of the datasets is unique for each dataset.

To maintain control over the I/O bandwidth allocated to a dataset, a given node is assigned to exactly one dataset, which means that the I/O bandwidth of that node is allocated solely to that dataset. We choose this approach for two reasons. First, in a large cluster, the node will often be an acceptable level of granularity. Second, performance insulation in storage clusters remains an open problem, and sharing nodes relies on it to be solved.

We define a dataset to be live at a given time if an application is reading or writing data of that dataset. The set of active nodes, $A(d)$, is the set of nodes that have been allocated to dataset $d$ and remain 'on'. The goal of I/O scheduling is, therefore, to allocate $A(d)$, for each of the datasets $d \in D_{L}$ where $D_{L}$ is the set of live datasets. Since a node can only be allocated to one dataset, an arbitration algorithm is required if multiple, live datasets store blocks on a particular node $i$. We make this choice, with one exception, by picking the dataset $d_{0}$, where $s_{i}\left(d_{0}\right)=\max s_{i}(d)$, with $d \in D_{i} \cap D_{L}$. That is we pick the live dataset that contributes the most to the score of the node. Compensation scores, in proportion to $s_{i}(d)$, are added to to all datasets $d \in D_{i} \cap D_{L}$ that were not chosen. The exception to this rule is when the dataset $d_{0}$ has the least compensation score among the datasets in $D_{i} \cap D_{L}$, in which case the dataset with the maximum compensation score is chosen. For instance, if all three datasets shown in Figure 7(a) are live, fair-sharing would set the active nodes of the datasets, as shown in Figure 7(b).

Rabbit and SpringFS can control the I/O bandwidth available to a dataset $d$ by controlling the size of the set $A(d)$. Since all requests for blocks belonging to that dataset are serviced by the nodes in the set $A(d)$, and no others, this sets the total amount of bandwidth available to the dataset. 


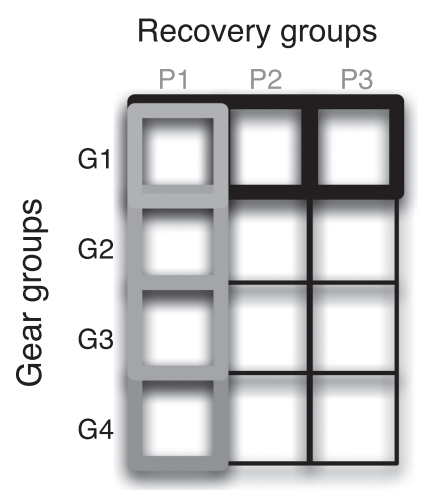

Fig. 8. Gear groups and recovery groups. All data from a single primary exists on a single recovery group, such as the grey box. When increasing the power setting, the file system turns on gear groups in an all-ornothing fashion.

Note that in addition to the capability of I/O scheduling, the multi-dataset mechanism also improves the capacity utilization of the systems, allowing small values of $p$ without limiting storage capacity utilization to $3 p / N$.

\subsection{Fault Tolerance}

This section describes modifications to the equal work layout that allow the file system to remain power proportional when a primary server fails. We only consider crash failures instead of arbitrary Byzantine failures. The failure recovery process is composed of three parts, though they are not necessarily separate activities. Each involves restoring some property of the file system.

- Availability. All data may be accessed immediately. In the case of a power proportional DFS, this means ensuring that every block is replicated on at least one active node.

- Durability. The file system's fault tolerance configuration is met. For Rabbit and SpringFS, this means that each block is replicated $r$ times.

- Layout. The file system's target layout is achieved. For the equal-work layout policy, non-primary node $i$ has approximately $B / i$ blocks on it.

Most of this discussion focuses on availability. Restoring durability and layout after a primary failure uses the same mechanisms as writing new data to the file system, described in Section 3.1.

The equal-work data layout cannot remain power proportional in the event that a primary server fails, because blocks from each primary server are scattered across all secondary servers. When a primary server fails, all secondary servers must be activated to restore availability. Therefore, the non-fault-tolerant version of the equalwork layout cannot achieve its target minimum power setting of $p$ when there is a primary server failure. Instead, it has a minimum power setting of $e p-1$.

To avoid this outcome, we impose further constraints on the secondary replicas of each block. The secondary servers are grouped into gear groups and recovery groups, with each server belonging to exactly one gear group and one recovery group. To visualize this, imagine arranging the secondary servers in a grid configuration depicted in Figure 8. The rows of this rectangle are the gear groups, and the columns are the recovery groups. The number of servers in these groups, that is, length of the rows and columns of the grid, are respectively known as the gear group size and recovery group size. 


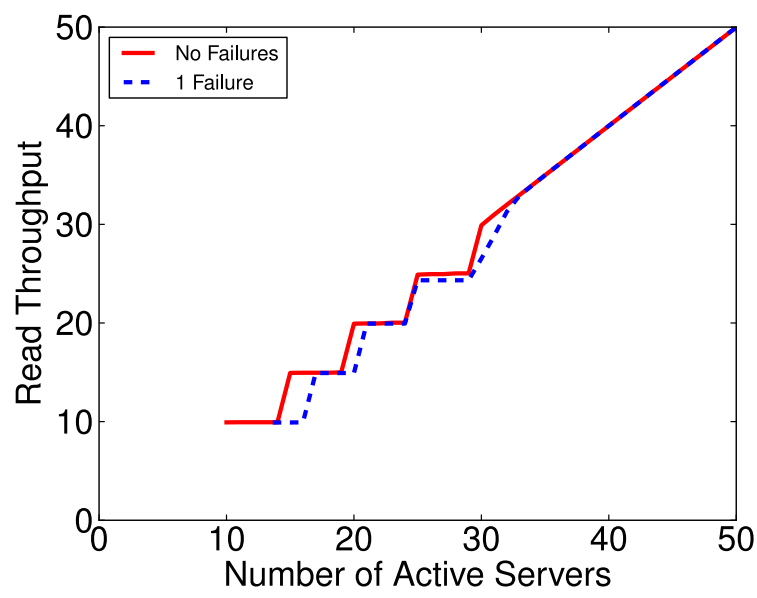

Fig. 9. Simulated performance of fault-tolerant layout with 10 primary servers and a gear size of 5 . The geared layout achieves very nearly ideal performance when a full gear is turned on, but less than ideal when a gear is only partially enabled.

Each primary server is mapped to exactly one recovery group. All data hosted by that primary server is replicated across its corresponding recovery group. This leads to a simple failure recovery strategy: if a primary server fails, the file system activates the corresponding recovery group. Because all of the server's secondary replicas reside in that recovery group, this is sufficient to restore availability to the data stored on the failed primary.

In this layout scheme, gear groups are the basic unit of power scaling. It is not helpful to turn on extra replicas for some primary server's data and not others: work can never be shared equally if some primaries have to read all of their blocks and others have to read only some of their blocks. Therefore, when turning on servers to increase the power mode, the file system must turn on a set of servers that will contain data from all primaries, that is, a gear group.

To share work equally, each gear group should contain approximately the same amount of data from each primary. The amount of data stored on each server in a gear group depends on where that gear group falls in the expansion chain. Servers belonging to low-numbered gear groups must store more data than those in high numbered gear groups, because they may be activated at lower-power modes. If the last server in a gear group is server number $i$, then every server in the gear group stores $\frac{B}{i}$ blocks. Equivalently, with a group size of $g$, each server in gear group $j$ stores $\frac{B}{(p+g j)}$ blocks.

Figure 9 shows the results of a simulation of the fault-tolerant layout in a failure-free case and with a single primary server failure. The performance is measured relative to the performance of a single server. The steps in the solid line show the effect of gearing: increasing the power setting causes no improvement in performance until a gear is completely activated, at which point the performance jumps up to the next level. The dotted line represents the power and performance curve in the case of a single primary failure. The file system can achieve the same performance with only a moderate increase in power.

This data layout creates a trade-off between gear size and recovery group size. A smaller gear size implies a larger recovery group size. By setting the gear size very small, we can achieve the goal of fine-grained power settings, but the large recovery group size means that in the event of a failure the minimum power setting will be high. 
Table I. Example Gear and Recovery Group Sizes

\begin{tabular}{|c|c|}
\hline Gear size & Recovery group size \\
\hline 1 & 174 \\
5 & 37 \\
10 & 20 \\
20 & 11 \\
50 & 6 \\
100 & 4 \\
\hline
\end{tabular}

Note: Even with a gear size of 5, allowing very fine grained scaling, the difference in minimum power setting is only $37 \%$.

On the other hand, a large gear size does not allow fine-grained power adjustments, but can run at very low power even when recovering from a failure. This relationship is complicated by the fact that the number of secondary servers depends on the gear size. Recall that the amount of data on each secondary server in gear $j$ is $\frac{B}{(p+g j)}$, meaning that choosing a larger gear group size causes less data to be stored on each server, thus requiring more secondary servers overall.

Table I shows the relationship between the gear size and recovery group for an example file system with 100 primary servers. The size of the recovery group, as a function of the number of primaries $p$ and the gear size $g$, is $\frac{e(p-g)-p}{g}$. As an example from this table, if the gear size is $10 \%$ of the number of primary servers, the recovery group size will be about $20 \%$ of the primary size. This means that the minimum power setting during failure recovery is only $20 \%$ higher than the minimum power setting with no failures. The ideal setting of these parameters depends on the workload of the file system and the rate of failure, but these results show that there is a wide range of reasonable settings for these parameters.

\section{IMPLEMENTATION}

SpringFS is implemented as a modified instance of the Hadoop Distributed File System (HDFS), version $0.19 .1 .^{7}$ We build on a Scriptable Hadoop interface that we built into Hadoop to allow experimenters to implement policies in external programs that are called by the modified Hadoop. This enables rapid prototyping of new policies for data placement, read load balancing, task scheduling, and rebalancing. It also enables us to emulate both Rabbit and SpringFS in the same system, for better comparison. SpringFS mainly consists of four components: data placement agent, load balancer, resizing agent, and migration agent-all implemented as python programs called by the Scriptable Hadoop interface.

Data Placement Agent. The data placement agent determines where to place blocks according to the SpringFS data layout. Ordinarily, when a HDFS client wishes to write a block, it contacts the HDFS NameNode and asks where the block should be placed. The NameNode returns a list of pseudorandomly chosen DataNodes to the client, and

\footnotetext{
${ }^{7}$ 0.19.1 was the latest Hadoop version when our work started. We have done a set of experiments to verify that HDFS performance differs little, on our experimental setup, between version 0.19 .1 and the latest stable version (1.2.1). We believe our results and findings are not significantly affected by still using this older version of HDFS.
} 
the client writes the data directly to these DataNodes. The data placement agent starts together with the NameNode and communicates with the NameNode using a simple text-based protocol over stdin and stdout. To obtain a placement decision for the $R$ replicas of a block, the NameNode writes the name of the client machine as well as a list of candidate DataNodes to the placement agent's stdin. The placement agent can then filter and reorder the candidates, returning a prioritized list of targets for the write operation. The NameNode then instructs the client to write to the first $R$ candidates returned.

Load Balancer. The load balancer implements the read offloading policy and preferentially sends reads to higher numbered servers that handle fewer write requests whenever possible. It keeps an estimate of the load on each server by counting the number of requests sent to each server recently. Every time SpringFS assigns a block to a server, it increments a counter for the server. To ensure that recent activity has precedence, these counters are periodically decayed by 0.95 every 5 seconds. While this does not give the exact load on each server, we find its estimates good enough (within $3 \%$ off optimal) for load balancing among relatively homogeneous servers.

Resizing Agent. The resizing agent changes SpringFS's footprint by setting an activity state for each DataNode. On every read and write, the data placement agent and load balancer will check these states and remove all "INACTIVE" DataNodes from the candidate list. Only "ACTIVE" DataNodes are able to service reads or writes. By setting the activity state for DataNodes, we allow the resources (e.g., CPU and network) of INACTIVE nodes to be used for other activities with no interference from SpringFS activities. We also modified the HDFS mechanisms for detecting and repairing underreplication to assume that INACTIVE nodes are not failed, so as to avoid undesired re-replication.

Migration Agent. The migration agent crawls the entire HDFS block distribution (once) when the NameNode starts, and it keeps this information up-to-date by modifying HDFS to provide an interface to get and change the current data layout. It exports two metadata tables from the NameNode, mapping file names to block lists and blocks to DataNode lists, and loads them into a SQLite database. Any changes to the metadata (e.g., creating a file, creating or migrating a block) are then reflected in the database on the fly. When data migration is scheduled, the SpringFS migration agent executes a series of SQL queries to detect layout problems, such as blocks with no primary replica or hosts storing too little data. It then constructs a list of migration actions to repair these problems. After constructing the full list of actions, the migration agent executes them in the background. To allow block-level migration, we modified the HDFS client utility to have a "relocate" operation that copies a block to a new server. The migration agent uses GNU Parallel to execute many relocates simultaneously.

\section{EVALUATION}

This section evaluates SpringFS and its offloading policies. Measurements of the SpringFS implementation show that it provide performance comparable to unmodified HDFS, that its policies improve agility by reducing the cleanup required, and that it can agilely adapt its number of active servers to provide required performance levels. In addition, analysis of six traces from real Hadoop deployments shows that SpringFS's agility enables significantly reduced commitment of active servers for the highly dynamic demands commonly seen in practice. 


\subsection{SpringFS prototype experiments}

Experimental Setup. Our experiments were run on a cluster of 31 machines. The modified Hadoop software is run within KVM virtual machines, for software management purposes, but each VM gets its entire machine and is configured to use all $8 \mathrm{CPU}$ cores, all 8GB RAM, and 100GB of local hard disk space. One machine was configured as the Hadoop master, hosting both the NameNode and the JobTracker. The other 30 machines were configured as slaves, each serving as an HDFS DataNode and a Hadoop TaskTracker. Unless otherwise noted, SpringFS was configured for 3-way replication $(R=3)$ and 4 primary servers $(p=4)$.

To simulate periods of high I/O activity, and effectively evaluate SpringFS under different mixes of I/O operations, we used a modified version of the standard Hadoop TestDFSIO storage system benchmark called TestDFSIO2. Our modifications allow for each node to generate a mix of block-size (128 MB) reads and writes, distributed randomly across the block ID space, with a user-specified write ratio.

Except where otherwise noted, we specify a file size of 2 GB per node in our experiments, such that the single Hadoop map task per node reads or writes 16 blocks. The total time taken to transfer all blocks is aggregated and used to determine a global throughput. In some cases, we break down the throughput results into the average aggregate throughput of just the block reads or just the block writes. This enables comparison of SpringFS's performance to the unmodified HDFS setup with the same resources.

Our experiments are focused primarily on the relative performance changes as agility-specific parameters and policies are modified. Because the original Hadoop implementation is unable to deliver the full performance of the underlying hardware, our system can only be compared reasonably with it and not the capability of the raw storage devices.

Effect of Offloading Policies. Our evaluation focuses on how SpringFS's offloading policies affect performance and agility. We also measure the cleanup work created by offloading and demonstrate that SpringFS's number of active servers can be adapted agilely to changes in workload intensity, allowing machines to be extracted and used for other activities.

Figure 10 presents the peak sustained I/O bandwidth measured for HDFS, Rabbit, and SpringFS at different offload settings. (Rabbit and SpringFS are identical when no offloading is used.) In this experiment, the write ratio is varied to demonstrate different mixes of read and write requests. SpringFS, Rabbit, and HDFS achieve similar performance for a read-only workload, because in all cases, there is a good distribution of blocks and replicas across the cluster over which to balance the load. The read performance of SpringFS slightly outperforms the original HDFS due to its explicit load tracking for balancing.

When no offloading is needed, both Rabbit and SpringFS are highly elastic and able to shrink $87 \%$ (26 non-primary servers out of 30) with no cleanup work. However, as the write workload increases, the equal-work layout's requirement that one replica be written to the primary set creates a bottleneck and eventually a slowdown of around $50 \%$ relative to HDFS for a maximum-speed write-only workload. SpringFS provides the flexibility to trade off some amount of agility for better write throughput under periods of high write load. As the write ratio increases, the effect of SpringFS's offloading policies becomes more visible. Using only a small number of offload servers, SpringFS significantly reduces the amount of data written to the primary servers and, as a result, significantly improves performance over Rabbit. For example, increasing the offload set from four (i.e., just the four primaries) to eight doubles maximum throughput 


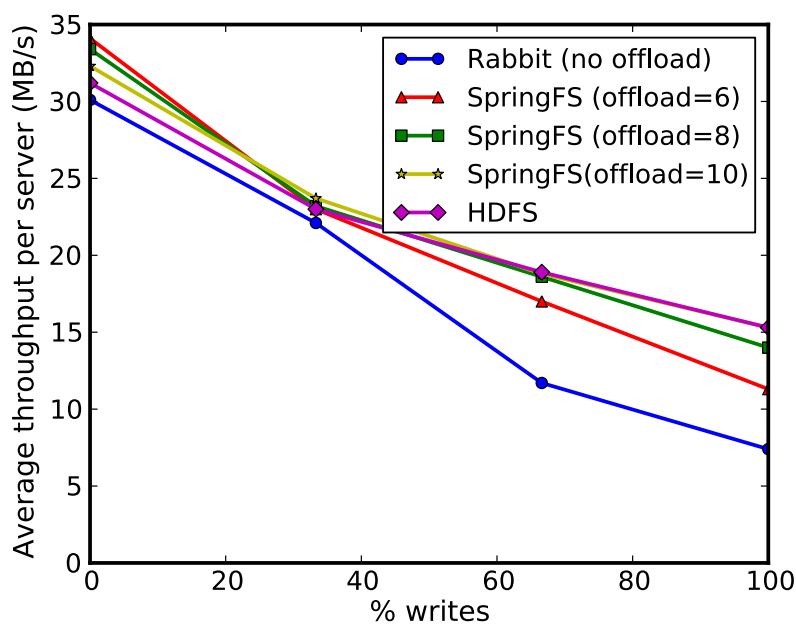

Fig. 10. Performance comparison of Rabbit with no offload, original HDFS, and SpringFS with varied offload set.

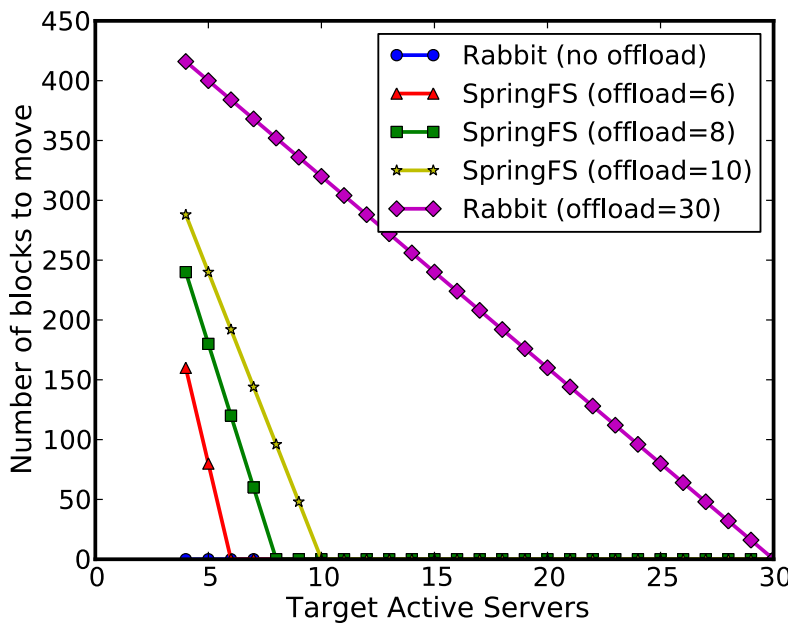

Fig. 11. Cleanup work (in blocks) needed to reduce active server count from 30 to X, for different offload settings. The (offload=6), (offload=8), and (offload=10) lines correspond to SpringFS with bounded write offloading. The (offload=30) line corresponds to Rabbit using Everest-style write offloading. Deactivating only non-offload servers requires no block migration. The amount of cleanup work is linear in the number of target active servers.

for the write-only workload, while remaining agile-the cluster is still able to shrink $74 \%$ with no cleanup work.

Figure 11 shows the number of blocks that need to be relocated to preserve data availability when reducing the number of active servers. As desired, SpringFS's data placements are highly amenable to fast extraction of servers. Shrinking the number of nodes to a count exceeding the cardinality of the offload set requires no clean-up work. Decreasing the count into the write offload set is also possible, but comes at some cost. As expected, for a specified target, the cleanup work grows with an increase in the offload target set. SpringFS with no offload reduces to the based equalwork layout, which needs no cleanup work when extracting servers but suffers from 


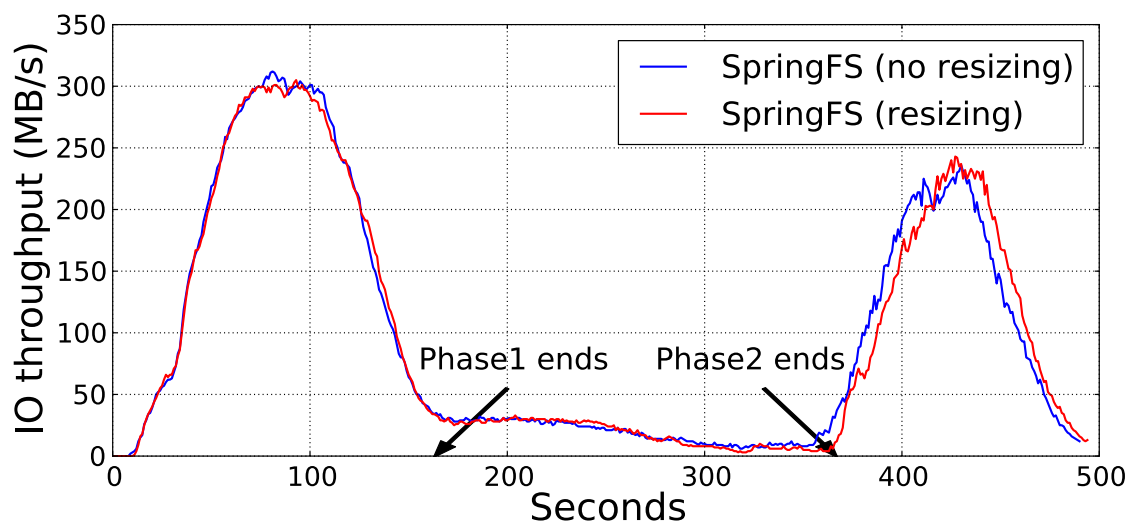

Fig. 12. Agile resizing in a 3-phase workload.

write performance bottlenecks. The most interesting comparison is Rabbit's full offload (offload=30) against SpringFS's full offload (offload=10). Both provide the cluster's full aggregate write bandwidth, but SpringFS's offloading scheme does it with much greater agility- $66 \%$ of the cluster could still be extracted with no cleanup work and more with small amounts of cleanup. We also measured actual cleanup times, finding (not surprisingly) that they correlate strongly with the number of blocks that must be moved.

SpringFS's read offloading policy is simple and reduces the cleanup work resulting from write offloading. To ensure that its simplicity does not result in lost opportunity, we compare it to the optimal, oracular scheduling policy with claircognizance of the HDFS layout. We use an Integer Linear Programming (ILP) model that minimizes the number of reads sent to primary servers from which primary replica writes are offloaded. The SpringFS read offloading policy, despite its simple realization, compares favorably and falls within $3 \%$ from optimal on average.

Agile Resizing in SpringFS. Figure 12 illustrates SpringFS's ability to resize quickly and deliver required performance levels. It uses a sequence of three benchmarks to create phases of workload intensity and measures performance for two cases: "SpringFS (no resizing)" where the full cluster stays active throughout the experiment and "SpringFS (resizing)" where the system size is changed with workload intensity. As expected, the performance is essentially the same for the two cases, with a small delay observed when SpringFS reintegrates servers for the third phase. However, the number of machine hours used is very different, as SpringFS extracts machines during the middle phase.

This experiment uses a smaller setup, with only 7 DataNodes, 2 primaries, 3 in the offload set, and 2-way replication. The workload consists of 3 consecutive benchmarks. The first benchmark is a TestDFSIO2 benchmark that writes 7 files, each $2 \mathrm{~GB}$ in size for a total of $14 \mathrm{~GB}$ written. The second benchmark is one SWIM job [Chen et al. 2011] randomly picked from a series of SWIM jobs synthesized from a Facebook trace which reads $4.2 \mathrm{~GB}$ and writes $8.4 \mathrm{~GB}$ of data. The third benchmark is also a TestDFSIO2 benchmark, but with a write ratio of $20 \%$. The TestDFSIO2 benchmarks are I/O intensive, whereas the SWIM job consumes only a small amount of the full I/O throughput. For the resizing case, 4 servers are extracted after the first write-only TestDFSIO2 benchmark finishes (shrinking the active set to 3), and those servers are reintegrated when the second TestDFSIO2 job starts. In this experiment, the resizing points are manually set when phase switch happens. Automatic resizing can be done 
Table II. Trace Summary

\begin{tabular}{|c|c|c|c|c|}
\hline Trace & Machines & Date & Length & $\begin{array}{c}\text { Bytes } \\
\text { processed }\end{array}$ \\
\hline CC-a & $<100$ & 2011 & 1 month & $69 \mathrm{~TB}$ \\
CC-b & 300 & 2011 & 9 days & $473 \mathrm{~TB}$ \\
CC-c & 700 & 2011 & 1 month & $13 \mathrm{~PB}$ \\
CC-d & $400-500$ & 2011 & 2.8 months & $5 \mathrm{~PB}$ \\
CC-e & 100 & 2011 & 9 days & $446 \mathrm{~TB}$ \\
FB & 3000 & 2010 & 10 days & $10.5 \mathrm{~PB}$ \\
\hline
\end{tabular}

CC is "Cloudera Customer" and FB is "Facebook". HDFS bytes processed is the sum of HDFS bytes read and HDFS bytes written.

based on previous work on workload prediction [Bodik et al. 2008; Gmach et al. 2007; Lin et al. 2011].

The results in Figure 12 are an average of 10 runs for both cases, shown with a moving average of 3 seconds. The I/O throughput is calculated by summing read throughput and write throughput multiplied by the replication factor. Decreasing the number of active SpringFS servers from 7 to 3 does not have an impact on its performance, since no cleanup work is needed. As expected, resizing the cluster from 3 nodes to 7 imposes a small performance overhead due to background block migration, but the number of blocks to be migrated is very small-about 200 blocks are written to SpringFS with only 3 active servers, but only 4 blocks need to be migrated to restore the equal-work layout. SpringFS's offloading policies keep the cleanup work small, for both directions. As a result, SpringFS extracts and reintegrates servers very quickly.

\subsection{Policy Analysis with Real-World Traces}

This section evaluates SpringFS in terms of machine-hour usage with real-world traces from six industry Hadoop deployments and compares it against three other storage systems: Rabbit, Sierra, and the default HDFS. We evaluate each system's layout policies with each trace, calculate the amount of cleanup work and the estimated cleaning time for each resizing action, and summarize the aggregated machine-hour usage consumed by each system for each trace. The results show that SpringFS significantly reduces machine-hour usage even compared to the state-of-the-art elastic storage systems, especially for write-intensive workloads.

Trace Overview. We use traces from six real Hadoop deployments representing a broad range of business activities, one from Facebook and five from different Cloudera customers. The six traces are described and analyzed in detail by Chen et al. [2012]. Table II summarizes key statistics of the traces. The Facebook trace (FB) comes from Hadoop DataNode logs, each record containing timestamp, operation type (HDFS_READ or HDFS_WRITE), and the number of bytes processed. From this information, we calculate the aggregate HDFS read/write throughput as well as the total throughput, which is the sum of read and write throughput multiplied by the replication factor ( 3 for all the traces). The five Cloudera customer traces (CC-a through CC-e, using the terminology from Chen et al. [2012]) all come from Hadoop job history logs, which contain per-job records of job duration, HDFS input/output size, etc. Assuming the amount of HDFS data read or written for each job is distributed evenly within the job duration, we also obtain the aggregated HDFS throughput at any given point of time, which is then used as input to the analysis program.

Trace Analysis and Results. To simplify calculation, we make several assumptions. First, the maximum measured total throughput in the traces corresponds to the 


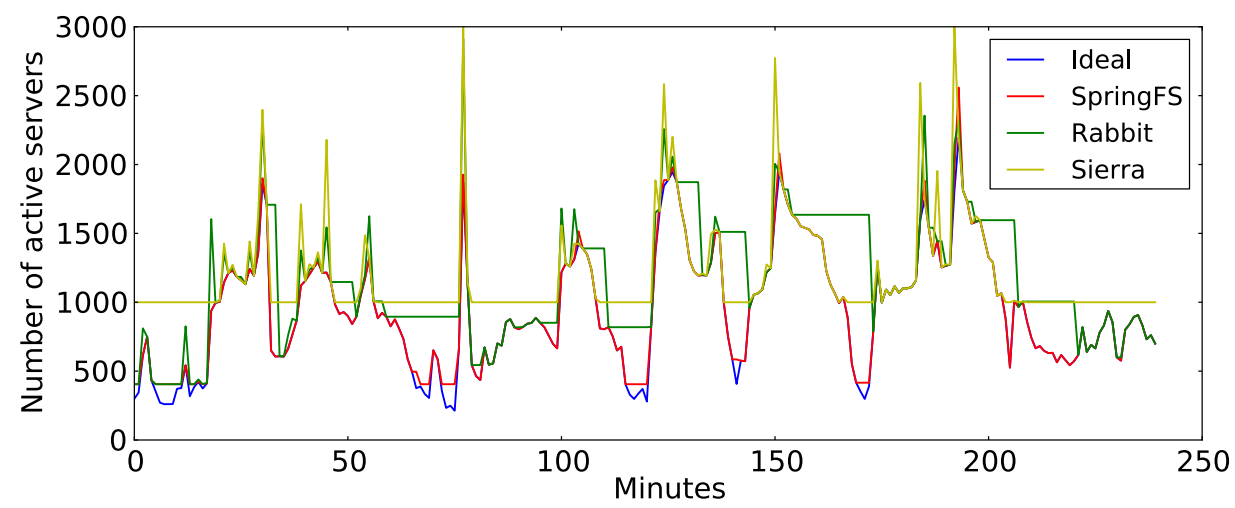

Fig. 13. Facebook trace.

maximum aggregate performance across all the machines in the cluster. Second, the maximum throughput a single machine can deliver, not differentiating reads and writes, is derived from the maximum measured total throughput divided by the number of machines in the cluster. In order to calculate the machine hour usage for each storage system, the analysis program needs to determine the number of active servers needed at any given point of time. It does this in the following steps: First, it determines the number of active servers needed in the imaginary "ideal" case, where no cleanup work is required at all, by dividing the total HDFS throughput by the maximum throughput a single machine can deliver. Second, it iterates through the number of active servers as a function of time. For each decrease in the active set of servers, it checks for any cleanup work that must be done by analyzing the data layout at that point. If any cleanup is required, it delays resizing until the work is done or the performance requirement demands an increase of the active set, to allow additional bandwidth for necessary cleanup work. For increases in the active set of servers, it turns on some extra servers to satisfy the read throughput and uses the extra bandwidth to do a fraction of migration work, using the passive migration policy (for all the systems) with the migration threshold set to be $\mathrm{T}=10$.

Figures 13 and 14 show the number of active servers needed, as a function of time, for the 6 traces. Each graph has 4 lines, corresponding to the "ideal" storage system, SpringFS, Rabbit, and Sierra, respectively. We do not show the line for the Default HDFS, but since it is not elastic, its curve would be a horizontal line with the number of active servers always being the full cluster size (the highest value on the Y-axis). While the original trace durations range from 9 days to 2.8 months, we only show a 4-hour-period for each trace for clarity. We start trace replaying more than 3 days before the 4-hour period, to make sure it represents the situation when systems are in a steady state and includes the effect of delaying migration work.

As expected, SpringFS exhibits better agility than Rabbit, especially when shrinking the size of the cluster, since it needs no cleanup work until resizing down to the offload set. Such agility difference between SpringFS and Rabbit is shown in Figure 13 at various points of time (e.g., at minute 110,140, and 160). The gap between the two lines indicates the number of machine hours saved due to the agility-aware read and bounded write policies used in SpringFS. SpringFS also achieves lower machine-hour usage than Sierra, as confirmed in all the analysis graphs. While a Sierra cluster can shrink down to $1 / 3$ of its total size without any cleanup work, it is not able to further decrease the cluster size. In contrast, SpringFS can shrink the cluster size down to approximately $10 \%$ of the original footprint. When $\mathrm{I} / \mathrm{O}$ activity is low, the difference 

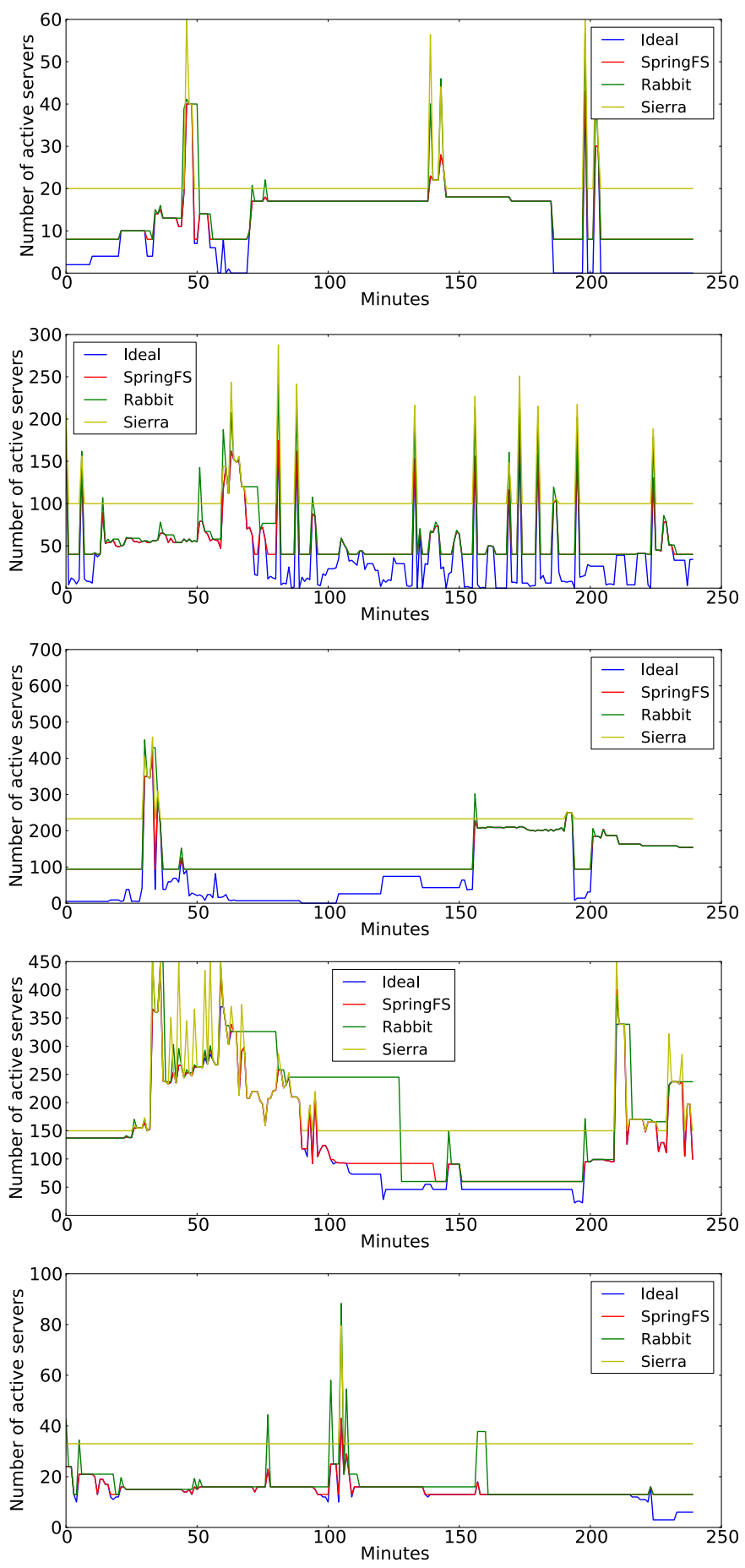

Fig. 14. Traces: CC-a, CC-b, CC-c, CC-d, and CC-e. 


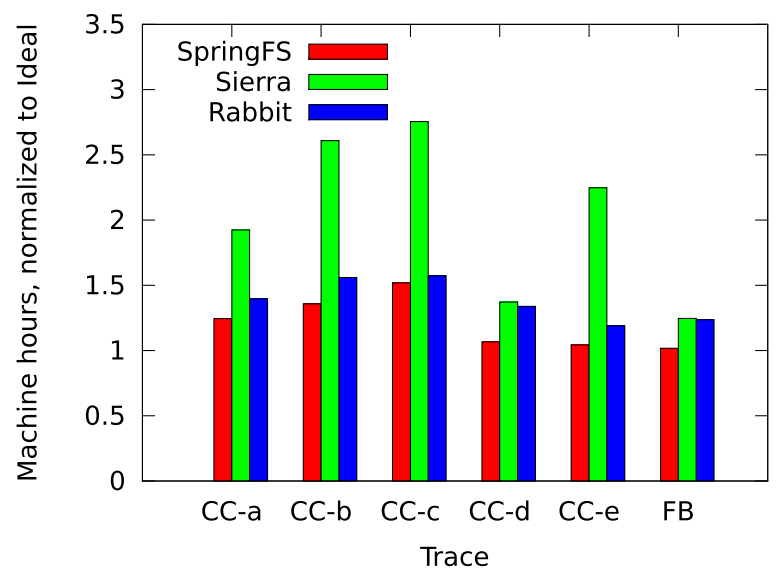

Fig. 15. Number of machine hours needed to execute each trace for each system, normalized to the "Ideal" system (1 on the y-axis, not shown).

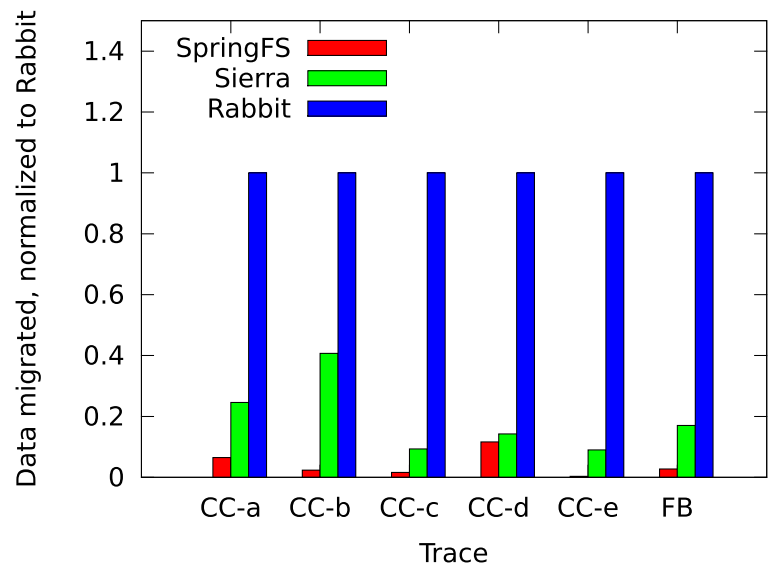

Fig. 16. Total data migrated for Rabbit, Sierra and SpringFS, normalized to results for Rabbit.

in minimal system footprint can have a significant impact on the machine-hour usage (e.g., as illustrated in Figure 14(b), Figure 14(c), and Figure 14(e)). In addition, when expanding cluster size, Sierra incurs more cleaning overhead than SpringFS, because deactivated servers need to migrate more data to restore its even data layout. These results are summarized in Figure 15, which shows the extra number of machine hours used by each storage system compared and normalized to the ideal system. In these traces, SpringFS outperforms the other systems by $6 \%$ to $120 \%$. For the traces with a relatively high write ratio, such as the FB, CC-d, and CC-e traces, SpringFS is able to achieve a "close-to-ideal" (within 5\%) machine-hour usage. SpringFS is less close to ideal for the other three traces because they frequently need even less than the $13 \%$ primary servers that SpringFS cannot deactivate.

Figure 16 summarizes the total amount of data migrated by Rabbit, Sierra, and SpringFS while running each trace. With bounded write offloading and read offloading, SpringFS is able to reduce the amount of data migration by a factor of 9-208, as compared to Rabbit. SpringFS migrates significantly less data than Sierra as well, 
because data migrated to restore the equal-work data layout is much less than that to restore an even data layout.

All of the trace analyses in the preceding text assume passive migration during server reintegration for all three systems compared, since it is useful to all of them. To evaluate the advantage of passive migration, specifically, we repeated the same trace analysis using the aggressive migration policy. The results show that passive migration reduces the amount of data migrated, relative to aggressive migration, by $1.5-7 \times$ (across the six traces) for SpringFS, $1.2-5.6 \times$ for Sierra, and 1.2-3 $\times$ for Rabbit. The benefit for Sierra and SpringFS is more significant, because their data migration occurs primarily during server reintegration.

\section{CONCLUSION}

SpringFS is a new elastic storage system that fills the space between state-of-the-art designs in the trade-off among agility, elasticity, and performance. SpringFS's data layout and offloading/migration policies adapt to workload demands and minimize the data redistribution cleanup work needed for elastic resizing, greatly increasing agility relative to the best previous elastic storage designs. As a result, SpringFS can satisfy the time-varying performance demands of real environments with many fewer machine hours. Such agility provides an important building block for resource-efficient data-intensive computing (a.k.a. Big Data) in multipurpose clouds with competing demands for server resources.

There are several directions for interesting future work. For example, the SpringFS data layout assumes that servers are approximately homogeneous, like HDFS does, but some real-world deployments end up with heterogeneous servers (in terms of I/O throughput and capacity) as servers are added and replaced over time. The data layout could be refined to exploit such heterogeneity, such as by using more powerful servers as primaries. Second, SpringFS's design assumes a relatively even popularity of data within a given dataset, as exists for Hadoop jobs processing that dataset, so it will be interesting to explore what aspects change when addressing the unbalanced access patterns (e.g., Zipf distribution) common in servers hosting large numbers of relatively independent files.

\section{ACKNOWLEDGMENTS}

We thank Cloudera and Facebook for sharing the traces and Yanpei Chen for releasing SWIM. We thank the members and companies of the PDL Consortium (including Actifio, APC, EMC, Facebook, Fusion-io, Google, HP Labs, Hitachi, Huawei, Intel, Microsoft Research, NEC Labs, NetApp, Oracle, Samsung, Seagate, Symantec, and Western Digital) for their interest, insights, feedback, and support.

\section{REFERENCES}

AMPLab. 2013. Algorithms, Machines, People Laboratory, Berkley. http://amplab.cs.berkeley.edu.

Hrishikesh Amur, James Cipar, Varun Gupta, Gregory R. Ganger, Michael A. Kozuch, and Karsten Schwan. 2010. Robust and flexible power-proportional storage. In Proceedings of the ACM Symposium on Cloud Computing. 217-228.

Peter Bodik, Michael Armbrust, Kevin Canini, Armando Fox, Michael Jordan, and David Patterson. 2008. A Case for Adaptive Datacenters to Conserve Energy and Improve reliability. University of California at Berkeley, Tech. Rep. UCB/EECS-2008-127.

Dhruba Borthakur. 2007. The Hadoop Distributed File System: Architecture and Design. The Apache Software Foundation.

Randal E. Bryant. 2007. Data-intensive supercomputing: The case for DISC. Tech. rep., Carnegie Mellon University.

Fay Chang, Jeffrey Dean, Sanjay Ghemawat,Wilson C. Hsieh, Deborah A.Wallach, Mike Burrows, Tushar Chandra, Andrew Fikes, and Robert E. Gruber. 2008. BigTable: A distributed storage system for structured data. ACM Trans. Comput. Syst. 26, 2, 1-26. 
Yanpei Chen, Sara Alspaugh, and Randy Katz. 2012. Interactive analytical processing in big data systems: A cross industry study of MapReduce workloads. Proc. VLDB Endow. 5, 12, 1802-1813.

Yanpei Chen, Archana Ganapathi, Rean Griffith, and Randy Katz. 2011. The case for evaluating MapReduce performance using workload suites. In Proceedings of the IEEE 9th International Symposium on Modeling, Analysis \& Simulation of Computer and Telecommunication Systems (MASCOTS).

Jeffrey Dean and Sanjay Ghemawat. 2008. MapReduce: Simplified data processing on large clusters. Commun. ACM 51, 1, 107-108.

Sanjay Ghemawat, Howard Gobioff, and Shun tak Leung. 2003. The Google file system. In Proceedings of the 19th ACM Symposium on Operating System Principles (SOSP). 29-43.

Daniel Gmach, Jerry Rolia, Ludmila Cherkasova, and Alfons Kemper. 2007. Workload analysis and demand prediction of enterprise data center applications. In Proceedings of the IEEE 10th International Symposium or Workload Characterization (IISWC).

Hadoop. 2012. The Apache Hadoop project. http://hadoop.apache.org.

Larry Hardesty. 2012. MIT, Intel unveil new initiatives addressing 'Big Data'. http://web.mit.edu/newsoffice/2012/big-data-csail-intel-center-0531.html.

Michael Isard, Vijayan Prabhakaran, Jon Currey, Udi Wieder, Kunal Talwar, and Andrew Goldberg. 2009. Quincy: Fair scheduling for distributed computing clusters. In Proceedings of the ACM SIGOPS 22nd Symposium on Operating Systems Principles (SOSP'09). ACM, New York, NY, 261-276.

ISTC-CC. 2013. Intel science and technology center - cloud computing. www.istc-cc.cmu.edu.

Jacob Leverich and Christos Kozyrakis. 2009. On the energy (in)efficiency of Hadoop clusters. In Proceedings of the Workshop on Power-Aware Computing and System HotPower.

Minghong Lin, AdamWierman, Lachlan L. H. Andrew, and Eno Thereska. 2011. Dynamic right-sizing for power-proportional data centers. In Proceedings of the INFOCOM.

Dushyanth Narayanan, Austin Donnelly, and Antony Rowstron. 2008a. Write off-loading: Practical power management for enterprise storage. In Proceedings of the USENIX Conference on File and Storage Technologies. USENIX Association, Berkeley, CA, 1-15.

Dushyanth Narayanan, Austin Donnelly, Eno Thereska, Sameh Elnikety, and Antony Rowstron. 2008b. Everest: Scaling down peak loads through I/O off-loading. In Proceedings of the 8th USENIX Symposium on Operating Systems and Implementation (OSD).

Yasushi Saito, Svend Frølund, Alistair Veitch, Arif Merchant, and Susan Spence. 2004. FAB: Building distributed enterprise disk arrays from commodity components. In Proceedings of the 11th International Conference on Architechtural Support for Programming Languages and Operating System. 48-58.

Eno Thereska, Austin Donnelly, and Dushyanth Narayanan. 2011. Sierra: Practical power-proportionality for data center storage. In Proceedings of the 6th Conference on Computer Systems (EuroSys). 169-182.

Nedeljko Vasić, Martin Barisits, Vincent Salzgeber, and Dejan Kostic. 2009. Making cluster applications energy-aware. In Proceedings of the Workshop on Automated Control for Datacenters and Clouds. 37-42.

CharlesWeddle, Mathew Oldham, Jin Qian, An-I AndyWang, Peter L. Reiher, and Geoffrey H. Kuenning. 2007. PARAID: A gear-shifting power-aware RAID. ACM Trans. Storage 3, 3, Article 13.

E. R. Zayas. 1991. AFS-3 programmer's reference: Architectural overview. Tech. Rep. Transarc Corporation.

Received August 2014; accepted September 2014 